\title{
REVIEW
}

\section{Transmission of antibiotic resistance genes in agroecosystems: an overview}

\author{
Jizheng HE (ه), Zhenzhen YAN, Qinglin CHEN \\ Faculty of Veterinary and Agricultural Sciences, The University of Melbourne, Parkville, Victoria 3010, Australia
}

\begin{abstract}
The use of antibiotics in human medicine and animal husbandry has resulted in the continuous release of antibiotics into the environment, which imposes high selection pressure on bacteria to develop antibiotic resistance. The spread and aggregation of antibiotic resistance genes (ARGs) in multidrug-resistant pathogens is one of the most intractable clinical challenges. Numerous studies have been conducted to profile the patterns of ARGs in agricultural ecosystems, as this is closely related to human health and wellbeing. This paper provides an overview of the transmission of ARGs in agricultural ecosystems resulting from the application of animal manures and other organic amendments. The future need to control and mitigate the spread of antibiotic resistance in agricultural ecosystems is also discussed, particularly from a holistic perspective, and requires multiple sector efforts to translate fundamental knowledge into effective strategies.
\end{abstract}

Keywords agroecosystem, antibiotic resistance, public health, soil-plant system

\section{Increasing prevalence of antibiotic resistance in the environment}

Antibiotics are medications that kill bacteria or slow down bacterial growth. Antibiotics have become central to modern healthcare since their introduction in the 1940s. From the 1950s, antibiotics have been approved by the U.S. Food and Drug Administration for use as growth promoters in food animals and to prevent and treat infections. The use or abuse of antibiotics for disease control or growth promotion in livestock husbandry, which comprises over $70 \%$ of the global consumption of

Received December 17, 2019; accepted January 22, 2020

Correspondence: jizheng.he@unimelb.edu.au antibiotics, has been widely reported to select and enrich for antibiotic resistant bacteria (ARB) in the gastrointestinal tracts of animals. Animal manures are therefore recognized as profuse sources of antibiotic residues, ARB and antibiotic resistance genes (ARGs) ${ }^{[1]}$. The emerging prevalence of antimicrobial resistance has put the advances of antibiotic usage at risk. The spread of ARB and ARGs represents a major threat to public health and may have potential risks for agricultural production and food security ${ }^{[2,3]}$ in the face of an almost complete absence of new antibiotics in development. The World Health Organization reports that the magnitude of antibiotic resistance has reached alarming levels in many countries, and calls on nations to develop a global action plan to combat antibiotic resistance. There is now compelling evidence that the environment constitutes a vast reservoir and important dissemination route of antibiotic resistance ${ }^{[4]}$. The majority of ARGs able to be acquired by human pathogens may have an environmental origin ${ }^{[5]}$. ARGs have therefore been recognized as a new type of environmental contaminant and the spread of antibiotic resistance in environmental settings is becoming one of the most intractable clinical challenges in the $21 \mathrm{st}$ century $^{[2]}$.

Increasing levels of ARGs have been reported at unprecedented rates in various environment settings, of which the spread of antibiotic resistance in agricultural ecosystems is a major concern because ARGs in agriculture are closely related to food safety and human health as illustrated in Fig. 1. There is a developing concern about the transmission of ARB and ARGs from agricultural settings to human pathogens through the food chain ${ }^{[6]}$. Increasing awareness and understanding of the pathways and mechanisms of ARG transmission across agricultural ecosystems is imperative for combating the looming public health threats posed by antibiotic resistance. Such knowledge is essential to the development of appropriate strategies and frameworks for tracking and management of the propagation of ARGs in the environment ${ }^{[7]}$. 


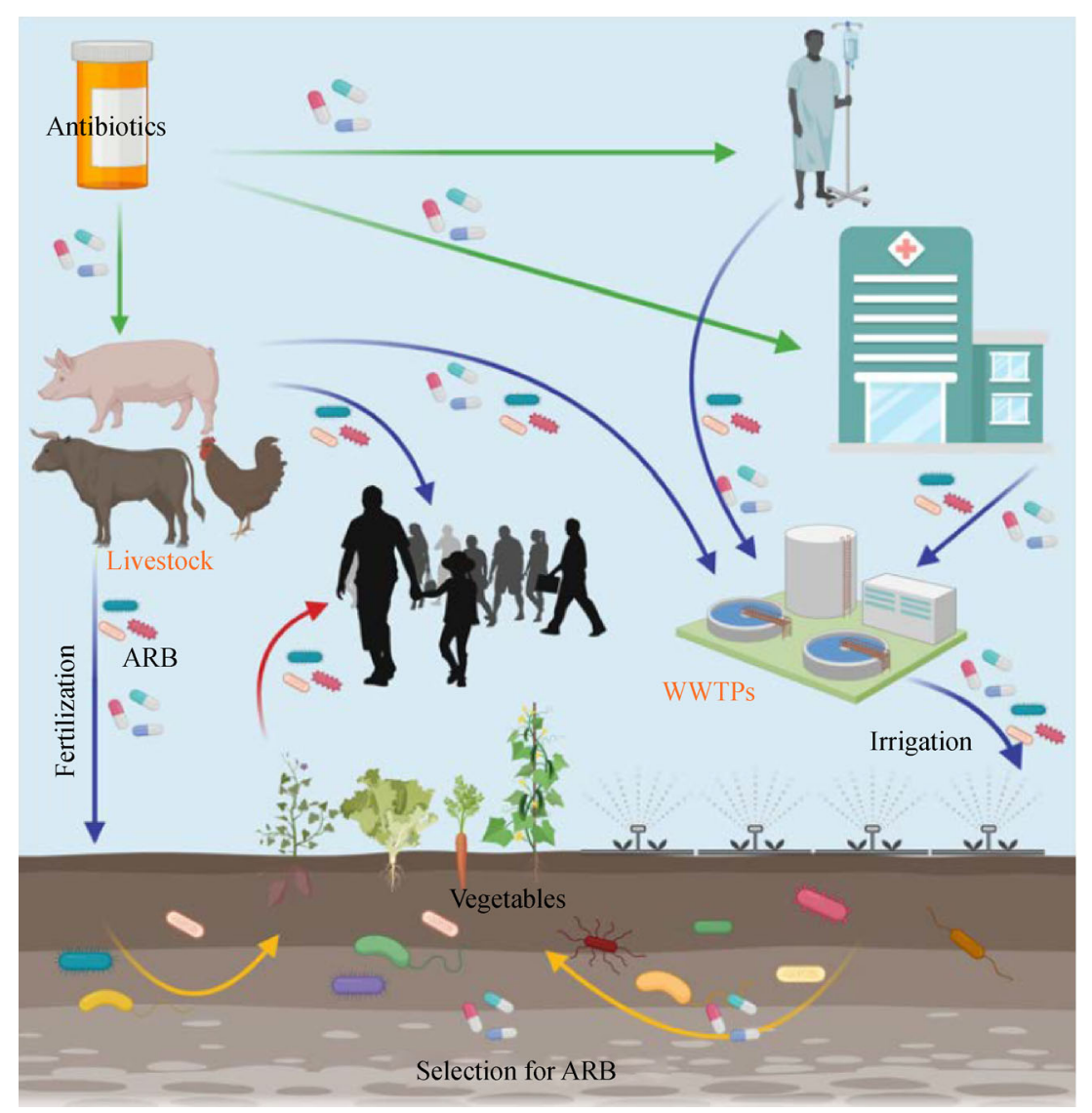

Fig. 1 Schematic diagram of the transmission of antibiotic resistance genes (ARGs) in agricultural ecosystems. Antibiotics can enter the soil through manure and sewage sludge application, and wastewater irrigation from wastewater treatment plants (WWTPs). The antibiotic residues can impose selection pressure on soil microbes and facilitate the dissemination of antibiotic resistance to vegetables. The plantassociated ARGs may represent a potential risk to human health, given the possibility of transfer between non-pathogenic antibiotic resistant bacteria (ARB) and human pathogens.

\section{Transmission of ARGs in agricultural soils}

Application of animal manures is a common practice for promoting crop production that represents a major pathway for environmental entry of considerable amounts of ARGs into agricultural soils and the dissemination of antibiotic resistance ${ }^{[8]}$. Increasing amounts of antibiotics are being used for disease treatment and growth promotion in animal husbandry, creating strong selection pressure on gut microbes which may lead to the proliferation of ARGs ${ }^{[9]}$. A substantial proportion (30\%-90\%) of antibiotics fed to farm animals are released into the environment through defecation and urination because the antibiotics are poorly metabolized $^{[10]}$. Manures are not only a profuse source of ARGs but can also promote the growth of potential ARGcarrying bacteria by providing nutrients ${ }^{[11]}$. Recent studies indicate that soils receiving animal manures are associated with high abundances of ARGs conferring resistance to some commonly used antibiotics, aminoglycosides, $\beta$ lactams and fluoroquinolones ${ }^{[12,13]}$. Processes such as using biochar for soil remediation and manure composting can greatly reduce the level and potential spread of ARGs but the risks of resistome transmission associated with manure application cannot be fully excluded ${ }^{[14]}$.

Reclaimed water irrigation and sewage sludge application represent another major pathway of the spread of ARGs to agricultural soils ${ }^{[15]}$. Municipal wastewaters are an important source of ARGs but current wastewater treatment processes cannot effectively remove antibiotics or ARGs, with the result that wastewater treatment plants (WWTPs) are major disseminators of antibiotic resistance. ARGs have been detected throughout the wastewater treatment process. Reclaimed water irrigation in urban environments is becoming more common due to rapid urbanization and water shortages. Irrigation with treated wastewater has been reported to lead to an enrichment of ARGs to thousands of times higher than in control soil ${ }^{[15]}$. In addition, the vast majority of wastewater-associated ARGs remain until the end of the treatment processes and are discharged in the form of sewage sludg $\mathrm{e}^{[16]}$. Land application of processed sewage sludges (e.g., organic fertilizers made from WWTP biosolids) as soil 
amendments is a key management approach for recycling plant nutrients from sewage but this represents a major pathway of ARG entry into agricultural ecosystems. The role of sewage sludge application in accelerating the dissemination of ARGs to soils through horizontal gene transfer has been shown in both a microcosm incubation experiment and a long-term field study ${ }^{[17]}$. More importantly, sewage sludge has been shown to have an abundance of bacteria carrying both ARGs and mobile genetic elements $^{[18]}$. Sewage sludge application may therefore enhance the spread of ARGs by horizontal transfer of mobile elements in soils and this may represent a potential risk to public health.

\section{Transmission of ARGs in the soll-plant system}

The enrichment of ARGs in agricultural soils has raised concerns because soil ARGs can be disseminated to plant microbiomes and lead to the potential spread of ARGs from farms to consumers ${ }^{[19]}$. Food consumption represents a major route for human exposure to ARGs in environmental microbiomes ${ }^{[20]}$. Some studies indicate that consumption of raw fruit and vegetable is a potential pathway for the dissemination of ARGs to human microbiomes $^{[21,22]}$.

It has been suggested that the long-term use of organic fertilizers causes ARG enrichment not only of soils but also of plant phyllosphere microbiomes ${ }^{[23]}$. Zhu et al. ${ }^{[24]}$ report that the abundance of ARGs in organically produced lettuce was about eight times higher than when chemical fertilizers were used, and Zhang et al. ${ }^{[6]}$ show that application of animal manure increased lettuce ARGs in both root endophytes and in the phyllosphere. A considerable proportion of ARGs in plant microbiomes overlapped with those in surrounding soil, indicating that soil might be a major source of plant ARGs ${ }^{[25]}$. There are many potential pathways by which soil-associated ARGs and ARB can transfer to plant microbiomes. ARG-carrying bacteria can (1) attach to the leaf surface through airborne particulates and survive as phyllosphere microorganisms, (2) colonize leaf tissue as endophytes, and/or (3) colonize roots as endophytes. In addition, plants can take up antibiotic residues from soils amended with manures and this may produce long-term selection pressure on the plant microbiome contributing to the emergence and spread of plant resistomes to the human food chain ${ }^{[20]}$. Furthermore, some ARGs have been detected in plant microbiomes but were not detectable in the surrounding environment, including soils, indicating an intrinsic ARG profile in plant microbiomes, which is important for establishing a baseline of the plant-associated antibiotic resistance before evaluating ARG transmission in the soil-plant system. Fortunately, most ARG-carrying bacteria in plant microbiomes are not pathogenic ${ }^{[21]}$.

\section{Future options to minimize the risks of antibiotic resistance}

It is now well recognized that the spread of antibiotic resistance is an increasing risk to public health. It is important to note that ARGs are a group of genes in a wide range of bacteria in the environment that are ancient and not harmful to humans unless they are transferred to human pathogens. Uncertainties therefore remain regarding the links between environmental ARGs and human health and further studies are required. It is imperative to understand the transmission pathways and mechanisms of ARGs in agricultural ecosystems. In addition, practical approaches should be taken to alleviate the spread of antibiotic resistance in the environment. First, the overuse or misuse of antibiotics in clinics and agriculture should be prevented, reducing antibiotic consumption. Second, organic manures and other organic amendments which are useful for replacing chemical fertilizers and provide biological benefits in controlling soilborne diseases and improving soil resilience should be pretreated to eliminate antibiotics and ARB. Third, the transmission of ARGs and $\mathrm{ARB}$ in agriculture should be monitored to assess their risk to food security and human health. In addition, guidelines for the application of organic amendments (organic fertilizers) and animal manures and sewage sludges should be developed. Using these approaches, control and mitigation of the spread of antibiotic resistance in agricultural ecosystems may be achieved together with the safe application of organic wastes to agricultural soils with a holistic perspective.

Acknowledgements This work was funded by the Australian Research Council (DP170103628) and the Australia-China Joint Research Centre project on Healthy Soils for Sustainable Healthy Food Production and Environmental Quality (ACSRF48165).

Compliance with ethics guidelines Jizheng $\mathrm{He}$, Zhenzhen Yan, and Qinglin Chen declare that they have no conflicts of interest or financial conflicts to disclose.

This article is a review and does not contain any studies with human or animal subjects performed by any of the authors.

\section{References}

1. Zhu Y G, Johnson T A, Su J Q, Qiao M, Guo G X, Stedtfeld R D, Hashsham S A, Tiedje J M. Diverse and abundant antibiotic resistance genes in Chinese swine farms. Proceedings of the National Academy of Sciences of the United States of America, 2013, 110(9): 3435-3440

2. World Health Organization (WHO). Antimicrobial resistance global report on surveillance: 2014 summary, World Health Organization: 2014. Available at WHO website on September 1, 2019

3. World Health Organization (WHO). Antimicrobial resistance. Available at WHO website on September 1, 2019

4. Bengtsson-Palme J, Kristiansson E, Larsson D G J. Environmental 
factors influencing the development and spread of antibiotic resistance. FEMS Microbiology Reviews, 2018, 42(1): 68-80

5. Forsberg K J, Reyes A, Wang B, Selleck E M, Sommer M O, Dantas G. The shared antibiotic resistome of soil bacteria and human pathogens. Science, 2012, 337(6098): 1107-1111

6. Zhang Y J, Hu H W, Chen Q L, Singh B K, Yan H, Chen D, He J Z. Transfer of antibiotic resistance from manure-amended soils to vegetable microbiomes. Environment International, 2019, 130: 104912

7. Ashbolt N J, Amézquita A, Backhaus T, Borriello P, Brandt K K, Collignon P, Coors A, Finley R, Gaze W H, Heberer T, Lawrence J R, Larsson D G, McEwen S A, Ryan J J, Schönfeld J, Silley P, Snape J R, Van den Eede C, Topp E. Human health risk assessment (HHRA) for environmental development and transfer of antibiotic resistance. Environmental Health Perspectives, 2013, 121(9): 993 1001

8. Udikovic-Kolic N, Wichmann F, Broderick N A, Handelsman J. Bloom of resident antibiotic-resistant bacteria in soil following manure fertilization. Proceedings of the National Academy of Sciences of the United States of America, 2014, 111(42): 15202 15207

9. Economou V, Gousia P. Agriculture and food animals as a source of antimicrobial-resistant bacteria. Infection and Drug Resistance, 2015, 8: 49-61

10. Zhang X X, Zhang T. Occurrence, abundance, and diversity of tetracycline resistance genes in 15 sewage treatment plants across China and other global locations. Environmental Science \& Technology, 2011, 45(7): 2598-2604

11. Hu H W, Wang J T, Li J, Li J J, Ma Y B, Chen D, He J Z. Fieldbased evidence for copper contamination induced changes of antibiotic resistance in agricultural soils. Environmental Microbiology, 2016, 18(11): 3896-3909

12. Hu H W, Han X M, Shi X Z, Wang J T, Han L L, Chen D, He J Z. Temporal changes of antibiotic-resistance genes and bacterial communities in two contrasting soils treated with cattle manure. FEMS Microbiology Ecology, 2016, 92(2): fiv169

13. Zhang Y J, Hu H W, Gou M, Wang J T, Chen D, He J Z. Temporal succession of soil antibiotic resistance genes following application of swine, cattle and poultry manures spiked with or without antibiotics. Environmental Pollution, 2017, 231(Pt 2): 1621-1632

14. Chen Q L, Fan X T, Zhu D, An X L, Su J Q, Cui L. Effect of biochar amendment on the alleviation of antibiotic resistance in soil and phyllosphere of Brassica chinensis L. Soil Biology and Biochemistry, 2018 119: 74-82
15. Wang F H, Qiao M, Su J Q, Chen Z, Zhou X, Zhu Y G. High throughput profiling of antibiotic resistance genes in urban park soils with reclaimed water irrigation. Environmental Science \& Technology, 2014, 48(16): 9079-9085

16. Bondarczuk K, Markowicz A, Piotrowska-Seget Z. The urgent need for risk assessment on the antibiotic resistance spread via sewage sludge land application. Environment International, 2016, 87: 4955

17. Chen Q L, An X L, Li H, Su J Q, Ma Y B, Zhu Y G. Long-term field application of sewage sludge increases the abundance of antibiotic resistance genes in soil. Environment International, 2016, 92-93: 110

18. Su J Q, Wei B, Ou-Yang W Y, Huang F Y, Zhao Y, Xu H J, Zhu Y G. Antibiotic resistome and its association with bacterial communities during sewage sludge composting. Environmental Science \& Technology, 2015, 49(12): 7356-7363

19. Looft T, Johnson T A, Allen H K, Bayles D O, Alt D P, Stedtfeld R D, Sul W J, Stedtfeld T M, Chai B, Cole J R, Hashsham S A, Tiedje J M, Stanton T B. In-feed antibiotic effects on the swine intestinal microbiome. Proceedings of the National Academy of Sciences of the United States of America, 2012, 109(5): 1691-1696

20. Chen Q L, Cui H L, Su J Q, Penuelas J, Zhu Y G. Antibiotic resistomes in plant microbiomes. Trends in Plant Science, 2019, 24 (6): 530-541

21. Zhang L, Kinkelaar D, Huang Y, Li Y, Li X, Wang H H. Acquired antibiotic resistance: are we born with it? Applied and Environmental Microbiology, 2011, 77(20): 7134-7141

22. Verraes C, Van Boxstael S, Van Meervenne E, Van Coillie E, Butaye P, Catry B, de Schaetzen M A, Van Huffel X, Imberechts H, Dierick K, Daube G, Saegerman C, De Block J, Dewulf J, Herman L. Antimicrobial resistance in the food chain: a review. International Journal of Environmental Research and Public Health, 2013, 10(7): 2643-2669

23. Chen Q L, An X L, Zheng B X, Ma Y B, Su J Q. Long-term organic fertilization increased antibiotic resistome in phyllosphere of maize. Science of The Total Environment, 2018, 645: 1230-1237

24. Zhu B K, Chen Q L, Chen S, Zhu Y G. Does organically produced lettuce harbor higher abundance of antibiotic resistance genes than conventionally produced? Environment International, 2017, 98: 152-159

25. Chen Q L, An X L, Zhu Y G, Su J Q, Gillings M R, Ye Z L, Cui L. Application of struvite alters the antibiotic resistome in soil, rhizosphere, and phyllosphere. Environmental Science \& Technology, 2017, 51(14): 8149-8157 